\title{
Jesús Martín Barbero y su condición de cartógrafo de América Latina: entrevista
}

\author{
Maria Patricia Téllez Garzón \\ Doctora; Universidad Javeriana, Bogotá, Colombia \\ maria.tellez@javeriana.edu.co
}

\begin{abstract}
Resumen
La entrevista parte de la tesis de doctorado del profesor Jesús Martín-Barbero titulada La palabra y la acción: por una dialéctica de la liberación, escrita en 1972 y publicada en libro recientemente, la cual sirve como punto de partida de su amplia e importante trayectoria académica. El punto clave en esa trayectoria fue su contacto e inmersión en América Latina a partir de su apropiación del lenguaje como herramienta de liberación - que toma prestado de Paulo Freire - y estrategia de mediación, y de su aproximación a lo popular como práctica comunicativa. Todo esto contribuye para afianzar su condición de cartógrafo de la comunicación.
\end{abstract}

\section{Palabras-clave}

Comunicación. Cartografía. Jesús Martín-Barbero. Entrevista.

Esta entrevista hace parte del homenaje que a finales de 2017 se le rindió en Porto Alegre (Brasil) a Jesús Martín Barbero en un evento respaldado por Centro Internacional de Estudios Superiores de Comunicación para América Latina (CIESPAL) y el Programa de PósGraduação em Comunicação e Informação de la Universidade Federal de Rio Grande do Sul (PPGCOM UFRGS) para conmemorar los 30 años de De los medios a las mediaciones (MARTÍN-BARBERO, 1987). En este encuentro se dieron cita investigadores latinoamericanos, seguidores de la obra de quien ha sido referente en los estudios de la comunicación en América Latina y cuyos aportes han sido definitivos para entender su realidad hoy.

Uno de los participantes en este evento, cuyo nombre se me escapa al escribir esta presentación, señaló como este había sido un ejercicio de memoria y afecto, y esta entrevista se mantiene en esta misma línea. Lo primero, en la medida en que fue una conversación para 
recuperar aspectos específicos de la importante trayectoria académica del homenajeado; y lo segundo por el agradecimiento de quienes hemos tenido la suerte y el honor de ser sus amigas, de conocerlo, trabajar con él y de formarnos académicamente a partir de sus conocimientos.

Maria Patricia Téllez Garzón: Vamos a iniciar esta conversación hablando de tu tesis de Doctorado en Filosofía ${ }^{1}$ (MARTÍN-BARBERO, 1972, 2018) que titulaste La palabra y la acción: por una dialéctica de la liberación, trabajo que como bien lo señalas en las múltiples entrevistas que te han hecho sirve como base y fundamento de tu recorrido académico. Allí en la introducción encontramos el interés por el tema del acontecimiento, de la política, de la acción y de la relación entre la teoría y la práctica en el continente.

Jesús Martín-Barbero: Para que veas que bien decía yo esas cosas. El primer parrafito de la tesis, que voy a leer, dice: “[...] los hombres viven y mueren al pie del acontecimiento. Y frente a las estructuras de opresión se alzan con el proyecto de liberación. El proyecto se encarna en una praxis que es palabra y acción indisolubles. La palabra explicita la conciencia que emerge de la acción y hecha pregunta horada la situación, su espesor macizo en apariencia, rompe el embrujo de la pasividad, diseña los contornos del camino. Solo la acción libera, pero habitada por la palabra nueva. Si la palabra sola es impotente la acción solo es estéril. La imagen del futuro se engendra entre las dos, la palabra. La palabra dibuja la utopía que las manos trabajan y el pedazo de tierra liberada hace verdad el poema." (MARTÍN-BARBERO, 2018, p. 3). Piensa que es el año 71 o $72 \ldots$

M.P.T: ¡Además de muy bonito, muy poético! ¿No?

J.M.B: ¡Oiga, yo soy poético!

M.P.T: Eso también lo aprendí leyendo sobre ti.

J.M.B: Hablemos entonces de la tesis. Yo titulé el prólogo Del saber con sabor y otros hallazgos pensantes, y en el primer párrafo, que es clave, digo lo siguiente: Para evitar confusiones digámoslo de entrada: el libro que tienes en las manos amigo lector, es una tesis de doctorado de Filosofía dirigida por el profesor Jean Ladrière, filósofo de la Ciencia, y presentado en la Universidad de Lovaina, Bélgica, en diciembre del año 1972. Así que lo primero con lo que te vas a topar es con la larga distancia del tiempo que va de los felices años 60 a los desazonados tiempos que atravesamos en el inicio del siglo, disque nuevo $u$ otro. Claro que en los nerviosos y destemplados tiempos que vivimos es mucha la gente que

\footnotetext{
11 El pasado 26 de abril se realizó el lanzamiento de la tesis en libro en el marco de la XXXI Feria Internacional de Bogotá y editada por el Centro Editorial Javeriano (CEJA).
} 
por contraste está convencida de que el mundo va bien. Entonces yo le voy a llevar la contraria a esa gente y para ello invito a mis lectores a dos caminatas: la primera es la del propio libro que tienes en las manos porque encontró la generosidad de un editor que habiéndolo leído decidió que el texto aún estaba vivo; y la segunda caminata es que, aunque escrito hace bastantes años no se trata de un libro viejo, ya que su verdadero tema es una visión latinoamericana del mundo.

M.P.T: Y es entonces cuando aparece América Latina y esto es muy importante. La pregunta que surge es ¿cómo te sumerges en el continente si venías de otro tan distante, y si además eras un filósofo y de repente empezaste a caminar por una realidad desconocida para ti en ese momento y te vuelves un cronista de ella buscando recorrer lo que llamas sus mapas nocturnos?

J.M.B: Esa es una buena pregunta. Tiene dos respuestas. La primera es que yo llego a Colombia el año 63, en octubre, y lo primero que hice al año siguiente fue dar clases en el Liceo Boston, un colegio privado en Bogotá y posteriormente me nombran director de la Biblioteca de Cultura Cristiana, a la que después llamo Centro Emmanuel Mounier, como un homenaje a uno de los más importantes filósofos cristianos de la época. La segunda razón es que Maria, mi hermana mayor, religiosa secular vivía en Chile hacia 20 años y yo quería ir a verla. Ella fue la primera que vino a América Latina y realmente tuvo una enorme influencia en mi decisión de venir a Colombia.

M.P.T: ¿Y en qué momento llegas a Chile? ¿Qué coyuntura política se vivía?

J.M.B: En Chile estuve dos meses y en ese momento estaba en las elecciones entre Frei, Allende y Durán. Inicialmente eran estos tres candidatos y después solo quedaron Allende y Frei. Y resulta que mi hermana me llevó a vivir en una casa de una familia con una abuela francesa de las que llevaron la Democracia Cristiana a ese país. Realmente fue una importación muy relevante en términos políticos. De tal manera que los debates entre Allende y Frei que duraban tres horas y después los ampliaban la abuela reunida en su casa con todos los hijos y los nietos. Todo esto en unas salas enormes que había en aquellos caserones al lado de la Catedral de Santiago, donde se debatía durante cuatro o cinco horas la diferencia entre lo que decían los de derecha que era "chilenizar" el cobre o nacionalizarlo. Lo primero es poético y lo segundo económico.

M.P.T: Una inmersión interesante a la realidad política de un país que iniciaba un proceso de trasformación donde participaba la iglesia, la clase obrera, los intelectuales, distintos sectores sociales interesados en el debate político. 
J.M.B: Es verdad. Entonces una semana me quedaba con los de Frei y la otra semana me iba a la callampa o barrio de invasión de Cisterna que así se llama una de las más grandes de la ciudad. Allí vivía mi hermana y trabajaba formando mujeres primero para no aguantarse al marido, segundo para hacer cosas que les retribuyeran a ellas y aprendieran a hacer cosas distintas a las que el marido le pedía. Y en ese lugar yo tuve una experiencia asombrosa porque de veras era eso. Una semana oyendo lo que pensaban los de centro derecha y otra los de centro izquierda o izquierda radical, era fantástico. 0 sea, podías ver y escuchar las dos versiones del mismo discurso. Y en medio de esa experiencia estuve dos meses. Eso fue todo aprendizaje y allí me hice latinoamericano.

M.P.T: ¿Y cuál fue la segunda experiencia que tuviste en ese proceso de ser Latinoamericano?

J.M.B: Esa experiencia chilena se complementó con un viajecito que pude hacer a Buenos Aires y a Montevideo. Pero donde disfruté realmente fue en esta última ciudad porque en la primera estaban las librerías, pero no tenía dinero para comprar libros. En la segunda había una familia de mi pueblo que estaba instalada allá, que era muy amiga de mi madre, y entonces yo le llevaba sus saludos y ellos me acogieron muy amablemente. Y lo lindo es que en ese lugar me encuentro con una escena culta, la gente está sentada, la familia y unos amigos, y todos en un debate larguísimo sobre el aborto, ¡Dios mío, en aquellos años! Un Jesuita, un ateo redomado y vieras la calidad de argumentos. Vea, yo dije, esto supera a Chile. Y es verdad, Uruguay superaba a Chile, ellos estaban más lejos, ellos habían parido una constitución expedida en el último mes del siglo XIX en la que no se habla de Dios. Una constitución laica, política. Bueno, entonces yo regresé de este viaje con un plus, o sea, si esto existe en el sur yo tengo que buscar en Colombia dónde está esto del sur. Entonces, muy bien. Allí empezó mi convicción de que yo no había llegado a Colombia, yo había llegado a América Latina.

J.M.B: Esta frase es textual y la utilicé igualmente en 2004 cuando recibí la nacionalidad colombiana. Yo hice un pequeño texto donde respondí con la frase más diplomática al acto político más político de mi vida. Allí después de agradecer a personas e instituciones termino con esta frase: "y gracias especiales a Colombia porque me hizo latinoamericano".

M.P.T: Me imagino pues para este momento ya tenías amigos en el continente y porque, por ejemplo, otro de tus afectos pasa por Brasil. Y pensando en esto yo te traje un regalo. Se trata de un ejemplar del libro Pedagogía del oprimido de Paulo Freire pues sé de 
tu admiración por este educador. Recuerdo que en una entrevista que te hacen Jorge Huergo y Kevin Morawicki (2016) en alguna parte tú dices que si a alguien tienes que agradecer en tu proceso de formación académica es a Paulo Freire y no a Marx.

J.M.B: Eso es verdad. Pero, oye, tú sabes que mi inspiración inicial fue Paul Ricouer quien fuera mi profesor y amigo, y a quien frecuenté a través de Jean Ladrière, el director de mi tesis. De alguna manera él es mi conexión con Freire a pesar de que él no lo conocía y esto para mí fue una gran decepción pues yo había trabajado un texto de este gran pedagogo latinoamericano. Para que Ricoeur lo entendiera tuve que explicarle sobre los planteamientos de este autor pues mucho de lo que yo estaba desarrollando en mi tesis estaba basado en él.

M.P.T: ¿Y cómo llegas a Paulo Freire?

J.M.B: A este último llego a través de un grupo de latinoamericanos liderado por José Abreu, un sociólogo y periodista brasileño, con quien se inicia en Paris un trabajo de reflexión sobre la realidad de América Latina que se materializa en el Servicio Europeo de Universitarios Latinoamericanos (SEUL) durante los años 1971 y 1972.

M.P.T: Volvamos a Freire como referente de tu tesis de Doctorado que de alguna manera se evidencia en el título La palabra y la acción: por una dialéctica de la liberación.

J.M.B: Yo encuentro a Freire inicialmente desde dos de sus trabajos: La Educación como práctica de la libertad y desde la Pedagogía del oprimido, publicados entre 1967 y 1968 y de modo específico a través de su compleja teoría del lenguaje. Posteriormente asisto a un ciclo de conferencias con las que inauguró su pequeño sitio que le abrieron en París. Este educador brasileño me dio las claves para desarrollar las tres partes de mi trabajo: la objetivación de la experiencia humana en el lenguaje, las múltiples posibilidades de su reapropiación $\mathrm{y}$, en un tercer momento, su potencialidad como espacio de emancipación y de liberación. Yo recupero el valor del lenguaje desde su espesor como mediador.

M.P.T: ¿Y el vínculo con la alfabetización como eje de reflexión de Freire?

J.M.B: En la primera parte de la tesis yo hago una síntesis de esa concepción del lenguaje donde Freire aporta algo nuevo para el pensamiento latinoamericano que consistía en ubicar la apropiación o reapropiación del lenguaje por los individuos y las comunidades como estrategias de mediación. Posteriormente a mi regreso a Colombia e inspirado una vez más en el método de alfabetización para adultos de este autor, con un amigo cuyo nombre 
no recuerdo en este momento, empezamos a desarrollar un trabajo de alfabetización muy interesante.

MPT: ¿En qué consistió el trabajo?

J.M.B: Con mi amigo empezamos en Bogotá y en Cartagena a enseñar a los viejos a leer y a escribir. Construyendo el universo de su barrio, el universo de palabra, ¿sí?, partiendo de una colección de palabras clave. Yo en Bogotá y él en Cartagena y fue una cosa incipiente, pero lo logramos. Logramos que fuera el universo de palabras fundadoras, de las palabras clave que movilizan el pensamiento de la mayoría de la gente. Entonces, primero tuve una experiencia desde la lectura de los textos de Paulo Freire y en un segundo momento los aplicamos para ver cómo funcionaban.

M.P.T: ¿Y la comunicación y su reflexión desde América Latina donde aparece?

J.M.B: Mi primera invitación a un congreso sobre comunicación se hizo con los montones de exiliados del sur que había en México. Y quién dirigió el congreso, el primer gran congreso de latinoamericanos, fue mi amigo Héctor Schmucler quien convoca a un seminario en la Universidad Autónoma Metropolitana, sede Xochimilco, que se llamó Encuentro Latinoamericano de Escuelas de Comunicación. Después, en Buenos Aires, Máximo Simpson editó desde Argentina las memorias en una publicación que tuvo una importante divulgación. Se trató de comunicación alternativa y cambio social en América Latina.

M.P.T: Y en esos eventos se fue dando el semillero de lo que serían los investigadores en comunicación en América Latina y las asociaciones que aparecen para agruparlos.

J.M.B: Es verdad. Y este hombre nos juntó en otro seminario un poco más tarde ya, donde estaban Javier Esteinou, Diego Portales, Fernando Reyes Matta, Oswaldo Capriles, Gustavo Esteva, Juan Gargurevich, Mario Kaplún, Daniel Prieto, Gregorio Selzer, el argentino y yo. Poco tiempo después yo fui a Buenos Aires para que armáramos lo que fue un intento de primer grupo de investigadores de Comunicación latinoamericano, y que tres años después se logró con la conformación definitiva de la Asociación Latinoamericana de Investigación en Comunicación (ALAIC).

J.M.B: En ese seminario en México, mientras todo el mundo hablaba de comunicación alternativa yo llego con esta apuesta de prácticas de comunicación en la cultura popular que luego se convirtió en el primer texto que yo hice en mi vida para publicar. El nombre digno es: Prácticas de comunicación en la cultura popular. En algún sitio debí poner: "plazas de 
mercado y cementerios". Ese era el título completo, pero a mi amigo le pareció demasiado largo.

M.P.T: ¿Y allí con este título de "Practicas de comunicación en la cultura popular" nace tu interés por este tema?

J.M.B: Yo creo que tú me has oído esa anécdota, pero ahora ya lo resumo. Lo popular es mi madre. Ella organizaba la economía de la casa y de mi pueblo en España, administrando una tienda de alimentos y ayudando a las familias pobres que en tiempos de la guerra no tenían otra posibilidad de comprar más allá del racionamiento. Ella murió cuando yo tenía 8 años y de ella, entre los 5 y los 8 años, yo aprendí del interés por los demás. Ella era una mujer de pueblo que rezaba a su manera pues en la iglesia se aburría de los sermones porque no los entendía. Cuando murió, todo el pueblo fue a su entierro como una forma de agradecer su generosidad.

M.P.T: Y por aquellas coincidencias la mayoría de estudiantes cuando te citan usan el Barbero como si fuera tu apellido paterno porque para ellos "Jesús Martin" se lo toman como si fuera un nombre.

J.M.B: Es verdad. Es algo mágico. Y es una forma de homenajearla.

M.P.T.: Volvamos a la historia sobre lo popular...

J.M.B: Cuando yo me largo de Colombia, porque estaba muy, muy cansado, y quería tomar distancia, quería además hacer un libro en serio. Vamos a decirlo con tono soberbio, un libro importante que ya tenía en la cabeza. Me tuve que ir un año a Madrid y los dos países que me ayudaron fueron Francia e Italia, y aprendí italiano no para hablarlo, pero sí para leerlo y eso fue mucho apoyo. Porque cuando yo estaba haciendo el Doctorado, como a los tres, cuatro meses llegó a la institución donde vivíamos un montón de latinoamericanos y españoles. Y un buen día un italiano se presenta en mi cuarto con los cuatro volúmenes de los textos de Gramsci, en italiano y un diccionario. Y me dijo, después de todo lo que hemos hablado, hablábamos cuando almorzábamos y cenamos, tú necesitas vitalmente leer a Gramsci. Y de modo particular a "Los cuadernos de la cárcel".

M.P.T: ¿Y en Colombia donde habías empezado a trabajar lo popular?

J.M.B: El tema de lo popular nace en la Universidad Jorge Tadeo Lozano donde me vinculo a la Escuela de Comunicación Social. Es muy importante. No es una querencia intelectual, es una querencia política. Yo empiezo a dar un curso de Comunicación en esa carrera y tenía muy claro que había que hacer una entrada, no digamos epistemológica, pero, particularmente sobre lo importante que es acceder al análisis de lo social, lo cultural y 
lo político. Era una cosa elemental. Yo empecé poniendo el lenguaje y la actuación. Entonces, ¿Qué es el lenguaje humano, la semiótica, la significación y la construcción de la significación? Y a partir de allí empezamos a mirar las prácticas de comunicación en los cementerios y en los supermercados cuyo texto se ha publicado montones de veces.

M.P.T: Y ya para cerrar Jesús, la última pregunta tiene que ver con tu percepción sobre nuestra realidad latinoamericana en esta coyuntura, ¿cómo ves esa relación entre la política, la comunicación, la ciudadanía y por supuesto los medios de comunicación?

J.M.B: Desencantada y desencantadora. Realmente no es solo América Latina. Cuando uno piensa desde donde yo pienso que son las relaciones entre la vida social, cultural y política, ¿cómo las culturas vivas, cotidianas, median entre las vidas sociales, las situaciones sociales y la política o lo político? Si partimos de esa pregunta nos encontramos con una mediación. Esta palabra aparece en la tesis unas diez mil veces. 0 sea, no es extraño que después apareciera dando el nombre de un libro. Porque verás, yo la usaba muchísimo en la tesis y tenía que ver eso con la ruptura de los dualismos maniqueos que se generaban en la política, en la ética, en el trabajo escolar, en el trabajo educativo. 0 buenos o malos, o listos o torpes. Todos esos dualismos que son los que han dominado la violencia, han manejado la violencia, han producido montones de violencia. Otra deuda pendiente en América Latina es el tema de la educación que no se acaba de resolver.

\section{A modo de cierre}

El pasado 26 de abril se realizó en el marco de la XXXI Feria Internacional de Bogotá el lanzamiento de la tesis La palabra y la acción: por una dialéctica de la liberación editada por la Editorial Pontificia Universidad Javeriana (MARTÍN-BARBERO, 2018). En este evento los interlocutores destacaron la vigencia de los planteamientos de Jesús Martín-Barbero, en su tesis, después de 46 años. Un cartógrafo es un hacedor de mapas y en esa tarea se ha mantenido hasta el momento Martín-Barbero. Diseñar nuevas cartografías para desde allí entender estos tiempos difíciles que vive el continente.

\section{Referências}

FREIRE, Paulo. Educação como prática da liberdade. Rio de Janeiro: Paz e Terra, 1967.

FREIRE, Paulo. Pedagogia do oprimido. Rio de Janeiro: Continuum, 1968.

HUERGO, Jorge; MORAWICI, Kewin. Memoria y promesa: conversaciones con Jesús Martin Barbero. La Plata: EDULP, 2016. 
MARTÍN-BARBERO, Jesús. De los medios a las mediaciones. Comunicación, cultura y hegemonia. Barcelona: Gustavo Gili, 1987.

MARTÍN-BARBERO, Jesús. La palabra y la acción: por una dialéctica de la liberación. 1972. Tesis (Doctorado em Filosofía) - Universidad Louvain, Louvain, 1972.

MARTÍN-BARBERO, Jesús. La palabra y la acción: por una dialéctica de la liberación. Bogotá: Editorial Javeriana, 2018.

\title{
Jesús Martín Barbero and his condition of cartographer of Latin America: interview
}

\begin{abstract}
The interview is part of the doctoral thesis entitled La palabra y la acción: por una dialéctica de la liberación, written by Professor Jesús Martín-Barbero in 1972 and published recently in a book. The publication serves as a starting point for his broad and important academic path. The key aspect in that trajectory was his contact and immersion in Latin America from his appropriation of language as a tool of freedom - borrowed from Paulo Freire - and mediation strategy and his approach to the popular as a communicative practice All this contributes to strengthen his status as a cartographer of communication.
\end{abstract}

\section{Keywords}

Communication. Cartography. Jesús Martín-Barbero. Interview.

\section{Jesús Martín Barbero e sua condição de cartógrafo da América Latina: entrevista}

\section{Resumo}

A entrevista parte da tese de doutorado do professor Jesús Martín-Barbero intitulada A palavra e a ação: por uma dialética da libertação (La palabra y la acción: por una dialéctica de la liberación, no original), escrita em 1972 e publicada recentemente em livro. $O$ ponto chave dessa trajetória foi seu contato e imersão em América Latina a partir de sua apropriação da linguagem como fermenta de libertação - que toma emprestado de Paulo Freire - e estratégia de mediação e de sua aproximação do popular como prática comunicativa. Tudo isso contribui para afiançar sua condição de cartógrafo da comunicação.

\section{Palavras-chave}

Comunicação. Cartografia. Jesús Martín-Barbero. Entrevista. 